\title{
Gamma-ray flares from the Crab Nebula
}

\author{
A. A. Abdo ${ }^{1}$, M. Ackermann ${ }^{2}$, M. Ajello ${ }^{2}$, A. Allafort ${ }^{2}$, L. Baldini ${ }^{3}$, J. Ballet ${ }^{4}$, G. Barbiellini ${ }^{5,6}$, \\ D. Bastieri ${ }^{7,8}$, K. Bechtol ${ }^{2}$, R. Bellazzini ${ }^{3}$, B. Berenji ${ }^{2}$, R. D. Blandford ${ }^{2, *}$, E. D. Bloom ${ }^{2}$, \\ E. Bonamente ${ }^{9,10}$, A. W. Borgland ${ }^{2}$, A. Bouvier ${ }^{2}$, T. J. Brandt ${ }^{11,12}$, J. Bregeon ${ }^{3}$, A. Brez ${ }^{3}$, \\ M. Brigida ${ }^{13,14}$, P. Bruel ${ }^{15}$, R. Buehler ${ }^{2, \dagger}$, S. Buson ${ }^{7,8}$, G. A. Caliandro ${ }^{16}$, R. A. Cameron ${ }^{2}$, \\ A. Cannon ${ }^{17,18}$, P. A. Caraveo ${ }^{19}$, J. M. Casandjian ${ }^{4}$, Ö. Çelik ${ }^{17,20,21}$, E. Charles ${ }^{2}$, A. Chekhtman ${ }^{22}$, \\ C. C. Cheung ${ }^{1}$, J. Chiang ${ }^{2}$, S. Ciprini ${ }^{10}$, R. Claus ${ }^{2}$, J. Cohen-Tanugi ${ }^{23}$, L. Costamante ${ }^{2}$, S. Cutini ${ }^{24}$, \\ F. D’Ammando ${ }^{25,26}$, C. D. Dermer ${ }^{27}$, A. de Angelis ${ }^{28}$, A. de Luca ${ }^{29}$, F. de Palma ${ }^{13,14}$, S. W. Digel ${ }^{2}$, \\ E. do Couto e Silva ${ }^{2}$, P. S. Drell ${ }^{2}$, A. Drlica-Wagner ${ }^{2}$, R. Dubois ${ }^{2}$, D. Dumora ${ }^{30}$, C. Favuzzi ${ }^{13,14}$, \\ S. J. Fegan ${ }^{15}$, E. C. Ferrara ${ }^{17}$, W. B. Focke ${ }^{2}$, P. Fortin ${ }^{15}$, M. Frailis ${ }^{28,31}$, Y. Fukazawa ${ }^{32}$, S. Funk ${ }^{2, \ddagger}$, \\ P. Fusco ${ }^{13,14}$, F. Gargano ${ }^{14}$, D. Gasparrini ${ }^{24}$, N. Gehrels ${ }^{17}$, S. Germani ${ }^{9,10}$, N. Giglietto ${ }^{13,14}$, \\ F. Giordano ${ }^{13,14}$, M. Giroletti ${ }^{33}$, T. Glanzman ${ }^{2}$, G. Godfrey ${ }^{2}$, I. A. Grenier ${ }^{4}$, M.-H. Grondin ${ }^{30}$, \\ J. E. Grove ${ }^{27}$, S. Guiriec ${ }^{34}$, D. Hadasch ${ }^{16}$, Y. Hanabata ${ }^{32}$, A. K. Harding ${ }^{17}$, K. Hayashi ${ }^{32}$, \\ M. Hayashida ${ }^{2}$, E. Hays ${ }^{17}$, D. Horan ${ }^{15}$, R. Itoh ${ }^{32}$, G. Jóhannesson ${ }^{35}$, A. S. Johnson ${ }^{2}$, T. J. Johnson ${ }^{17,36}$, \\ D. Khangulyan ${ }^{42}$, T. Kamae ${ }^{2}$, H. Katagiri ${ }^{32}$, J. Kataoka ${ }^{37}$, M. Kerr ${ }^{38}$, J. Knödlseder ${ }^{11}$, M. Kuss ${ }^{3}$, \\ J. Lande ${ }^{2}$, L. Latronico ${ }^{3}$, S.-H. Lee ${ }^{2}$, M. Lemoine-Goumard ${ }^{30}$, F. Longo ${ }^{5,6}$, F. Loparco ${ }^{13,14}$, \\ P. Lubrano ${ }^{9,10}$, G. M. Madejski ${ }^{2}$, A. Makeev ${ }^{22}$, M. Marelli ${ }^{19}$, M. N. Mazziotta ${ }^{14}$, J. E. McEnery ${ }^{17,36}$, \\ P. F. Michelson ${ }^{2}$, W. Mitthumsiri ${ }^{2}$, T. Mizuno ${ }^{32}$, A. A. Moiseev ${ }^{20,36}$, C. Monte ${ }^{13,14}$, M. E. Monzani ${ }^{2}$, \\ A. Morselli ${ }^{39}$, I. V. Moskalenko ${ }^{2}$, S. Murgia ${ }^{2}$, T. Nakamori ${ }^{37}$, M. Naumann-Godo ${ }^{4}$, P. L. Nolan ${ }^{2}$, \\ J. P. Norris ${ }^{40}$, E. Nuss ${ }^{23}$, T. Ohsugi ${ }^{41}$, A. Okumura ${ }^{42}$, N. Omodei ${ }^{2}$, J. F. Ormes ${ }^{40}$, M. Ozaki ${ }^{42}$, \\ D. Paneque $^{2}$, D. Parent ${ }^{22}$, V. Pelassa ${ }^{23}$, M. Pepe ${ }^{9,10}$, M. Pesce-Rollins ${ }^{3}$, M. Pierbattista ${ }^{4}$, F. Piron ${ }^{23}$, \\ T. A. Porter ${ }^{2}$, S. Rainò ${ }^{13,14}$, R. Rando ${ }^{7,8}$, P. S. Ray ${ }^{27}$, M. Razzano ${ }^{3}$, A. Reimer ${ }^{43,2}$, O. Reimer ${ }^{43,2}$, \\ T. Reposeur ${ }^{30}$, S. Ritz ${ }^{44}$, R. W. Romani ${ }^{2}$, H. F.-W. Sadrozinski ${ }^{44}$, D. Sanchez ${ }^{15}$, P. M. Saz Parkinson ${ }^{44}$, \\ J. D. Scargle ${ }^{45}$, T. L. Schalk ${ }^{44}$, C. Sgrò ${ }^{3}$, E. J. Siskind ${ }^{46}$, P. D. Smith ${ }^{12}$, G. Spandre ${ }^{3}$, P. Spinelli ${ }^{13,14}$, \\ M. S. Strickman ${ }^{27}$, D. J. Suson ${ }^{47}$, H. Takahashi ${ }^{41}$, T. Takahashi ${ }^{42}$, T. Tanaka ${ }^{2}$, J. B. Thayer ${ }^{2}$, \\ D. J. Thompson ${ }^{17}$, L. Tibaldo ${ }^{7,8,4,48}$, D. F. Torres ${ }^{16,49}$, G. Tosti ${ }^{9,10}$, A. Tramacere ${ }^{2,50,51}$, E. Troja ${ }^{17,52}$, \\ Y. Uchiyama ${ }^{2}$, J. Vandenbroucke ${ }^{2}$, V. Vasileiou ${ }^{20,21}$, G. Vianello ${ }^{2,50}$, V. Vitale ${ }^{39,53}$, P. Wang ${ }^{2}$, \\ K. S. Wood $^{27}$, Z. Yang ${ }^{54,55}$, M. Ziegler ${ }^{44}$
}

1. National Research Council Research Associate, National Academy of Sciences, Washington, DC 20001, resident at Naval Research Laboratory, Washington, DC 20375, USA

2. W. W. Hansen Experimental Physics Laboratory, Kavli Institute for Particle Astrophysics 
and Cosmology, Department of Physics and SLAC National Accelerator Laboratory, Stanford University, Stanford, CA 94305, USA

3. Istituto Nazionale di Fisica Nucleare, Sezione di Pisa, I-56127 Pisa, Italy

4. Laboratoire AIM, CEA-IRFU/CNRS/Université Paris Diderot, Service d'Astrophysique, CEA Saclay, 91191 Gif sur Yvette, France

5. Istituto Nazionale di Fisica Nucleare, Sezione di Trieste, I-34127 Trieste, Italy

6. Dipartimento di Fisica, Università di Trieste, I-34127 Trieste, Italy

7. Istituto Nazionale di Fisica Nucleare, Sezione di Padova, I-35131 Padova, Italy

8. Dipartimento di Fisica “G. Galilei”, Università di Padova, I-35131 Padova, Italy

9. Istituto Nazionale di Fisica Nucleare, Sezione di Perugia, I-06123 Perugia, Italy

10. Dipartimento di Fisica, Università degli Studi di Perugia, I-06123 Perugia, Italy

11. Centre d'Étude Spatiale des Rayonnements, CNRS/UPS, BP 44346, F-30128 Toulouse Cedex 4, France

12. Department of Physics, Center for Cosmology and Astro-Particle Physics, The Ohio State University, Columbus, OH 43210, USA

13. Dipartimento di Fisica "M. Merlin" dell’Università e del Politecnico di Bari, I-70126 Bari, Italy

14. Istituto Nazionale di Fisica Nucleare, Sezione di Bari, 70126 Bari, Italy

15. Laboratoire Leprince-Ringuet, École polytechnique, CNRS/IN2P3, Palaiseau, France

16. Institut de Ciencies de l'Espai (IEEC-CSIC), Campus UAB, 08193 Barcelona, Spain

17. NASA Goddard Space Flight Center, Greenbelt, MD 20771, USA

18. University College Dublin, Belfield, Dublin 4, Ireland

19. INAF-Istituto di Astrofisica Spaziale e Fisica Cosmica, I-20133 Milano, Italy

20. Center for Research and Exploration in Space Science and Technology (CRESST) and NASA Goddard Space Flight Center, Greenbelt, MD 20771, USA

21. Department of Physics and Center for Space Sciences and Technology, University of Maryland Baltimore County, Baltimore, MD 21250, USA 
22. College of Science, George Mason University, Fairfax, VA 22030, resident at Naval Research Laboratory, Washington, DC 20375, USA

23. Laboratoire de Physique Théorique et Astroparticules, Université Montpellier 2, CNRS/IN2P3, Montpellier, France

24. Agenzia Spaziale Italiana (ASI) Science Data Center, I-00044 Frascati (Roma), Italy

25. IASF Palermo, 90146 Palermo, Italy

26. INAF-Istituto di Astrofisica Spaziale e Fisica Cosmica, I-00133 Roma, Italy

27. Space Science Division, Naval Research Laboratory, Washington, DC 20375, USA

28. Dipartimento di Fisica, Università di Udine and Istituto Nazionale di Fisica Nucleare, Sezione di Trieste, Gruppo Collegato di Udine, I-33100 Udine, Italy

29. Istituto Universitario di Studi Superiori (IUSS), I-27100 Pavia, Italy

30. Université Bordeaux 1, CNRS/IN2p3, Centre d'Études Nucléaires de Bordeaux Gradignan, 33175 Gradignan, France

31. Osservatorio Astronomico di Trieste, Istituto Nazionale di Astrofisica, I-34143 Trieste, Italy

32. Department of Physical Sciences, Hiroshima University, Higashi-Hiroshima, Hiroshima 739-8526, Japan

33. INAF Istituto di Radioastronomia, 40129 Bologna, Italy

34. Center for Space Plasma and Aeronomic Research (CSPAR), University of Alabama in Huntsville, Huntsville, AL 35899, USA

35. Science Institute, University of Iceland, IS-107 Reykjavik, Iceland

36. Department of Physics and Department of Astronomy, University of Maryland, College Park, MD 20742, USA

37. Research Institute for Science and Engineering, Waseda University, 3-4-1, Okubo, Shinjuku, Tokyo, 169-8555 Japan

38. Department of Physics, University of Washington, Seattle, WA 98195-1560, USA

39. Istituto Nazionale di Fisica Nucleare, Sezione di Roma "Tor Vergata", I-00133 Roma, Italy

40. Department of Physics and Astronomy, University of Denver, Denver, CO 80208, USA 
41. Hiroshima Astrophysical Science Center, Hiroshima University, Higashi-Hiroshima, Hiroshima 739-8526, Japan

42. Institute of Space and Astronautical Science, JAXA, 3-1-1 Yoshinodai, Chuo-ku, Sagamihara, Kanagawa 252-5210, Japan

43. Institut für Astro- und Teilchenphysik and Institut für Theoretische Physik, LeopoldFranzens-Universität Innsbruck, A-6020 Innsbruck, Austria

44. Santa Cruz Institute for Particle Physics, Department of Physics and Department of Astronomy and Astrophysics, University of California at Santa Cruz, Santa Cruz, CA 95064, USA

45. Space Sciences Division, NASA Ames Research Center, Moffett Field, CA 94035-1000, USA

46. NYCB Real-Time Computing Inc., Lattingtown, NY 11560-1025, USA

47. Department of Chemistry and Physics, Purdue University Calumet, Hammond, IN 463232094, USA

48. Partially supported by the International Doctorate on Astroparticle Physics (IDAPP) program

49. Institució Catalana de Recerca i Estudis Avançats (ICREA), Barcelona, Spain

50. Consorzio Interuniversitario per la Fisica Spaziale (CIFS), I-10133 Torino, Italy

51. INTEGRAL Science Data Centre, CH-1290 Versoix, Switzerland

52. NASA Postdoctoral Program Fellow, USA

53. Dipartimento di Fisica, Università di Roma "Tor Vergata", I-00133 Roma, Italy

54. Department of Physics, Stockholm University, AlbaNova, SE-106 91 Stockholm, Sweden

55. The Oskar Klein Centre for Cosmoparticle Physics, AlbaNova, SE-106 91 Stockholm, Sweden

† buehler@stanford.edu,*rdb3@stanford.edu, łfunk@ slac.stanford.edu 
A young and energetic pulsar powers the well-known Crab Nebula. Here we describe two separate gamma-ray (photon energy $>100 \mathrm{MeV}$ ) flares from this source detected by the Large Area Telescope on board the Fermi Gammaray Space Telescope. The first flare occurred in February 2009 and lasted approximately 16 days. The second flare was detected in September 2010 and lasted approximately 4 days. During these outbursts the gamma-ray flux from the nebula increased by factors of four and six, respectively. The brevity of the flares implies that the gamma rays were emitted via synchrotron radiation from $\mathrm{PeV}\left(10^{15} \mathrm{eV}\right)$ electrons in a region smaller than $1.4 \times 10^{-2} \mathrm{pc}$. These are the highest energy particles that can be associated with a discrete astronomical source, and they pose challenges to particle acceleration theory.

The Crab Nebula is the remnant of an historical supernova (SN), recorded in 1054 C.E., located at a distance of $2 \mathrm{kpc}(1)$. The $\mathrm{SN}$ explosion left behind a pulsar, which continuously emits a wind of magnetized plasma of electron/positron pairs (henceforth referred to as electrons). This pulsar wind is expected to terminate in a standing shock where the particles may undergo shock acceleration $(2,3)$. As the electrons diffuse into the downstream medium they release energy through interactions with the surrounding magnetic and photon fields. This emission is observed across all wavebands from radio up to TeV gamma-ray energies and is referred to as a pulsar wind nebula (PWN). The efficiency of this process is remarkable. As much as $30 \%$ of the total energy released by the Crab pulsar is emitted by the PWN [ (4) and references therein]. The Crab PWN has an approximately ellipsoidal shape on the sky with a size that decreases with increasing photon energy. At radio frequencies it extends out to $5^{\prime}(3 \mathrm{pc})$ from the central pulsar. At X-ray wavelengths a bright torus surrounds the pulsar; its radius is $40^{\prime \prime}$ $(0.4 \mathrm{pc})$ and jets emerge perpendicular to it in both directions.

Within the region encapsulated by the torus there are several small-scale structures. The inner nebula, which we define as the central $15^{\prime \prime}$ around the pulsar, has several small-scale regions of variable X-ray and optical brightness. The most prominent is an X-ray-bright inner ring with a radius of $10^{\prime \prime}(0.1 \mathrm{pc})$; this ring is thought to represent the termination shock of the PWN (5). Several knots with diameters of $\sim 1^{\prime \prime}(0.01 \mathrm{pc})$ are detected close to the inner ring and the base of the jets, and bright arcs of comparable width are observed moving outwards from the inner ring into the torus $(6,7)$.

The broad-band spectral energy distribution (SED) of the Crab Nebula is composed of two broad non-thermal components. A low-energy component dominates the overall output and extends from radio to gamma-ray frequencies. This emission is thought to be from synchrotron radiation. This notion is confirmed in radio to X-ray frequencies with polarization measurements $(8-10)$. The emission of this synchrotron component peaks between optical and X-ray frequencies, where the emission is primarily from the torus (5). The emission site of higherenergy photons (beyond $100 \mathrm{keV}$ ) cannot be resolved due to the limited angular resolution of telescopes observing at these frequencies. The high-energy component dominates the emission above $\sim 400 \mathrm{MeV}$ and is thought to be emitted via inverse Compton (IC) scattering, predominantly of the synchrotron photons $(11,12)$. 
The large-scale integrated emission from the Crab Nebula is expected to be steady within a few percent and is thus often used to cross-calibrate X-ray and gamma-ray telescopes and to check their stability over time $(13,14)$. Recently, variability in the x-ray flux from the nebula by $\sim 3.5 \% \mathrm{yr}^{-1}$ has been detected, setting limits on the accuracy of this practice (15). Yearly variations in the emission in the high-energy tail (1-150 MeV) of the synchrotron component has also been reported $(16,17)$. No significant variations have been detected for the high-energy component of the nebula (18-20).

The Large Area Telescope (LAT) on board the Fermi Gamma-Ray Space Telescope (Fermi) has continuously monitored the Crab Nebula as a part of its all-sky survey since August 2008. The LAT detects gamma rays from $20 \mathrm{MeV}$ to $>300 \mathrm{GeV}$, and this spans the transition region between the low and the high-energy components of the nebular spectrum. The average SED measured during the first 25 months of observations (Fig. 1) is well characterized by the sum of two spectral components, each with a power-law dependence on energy (21). The integrated flux of the low-energy component is $(6.2 \pm 0.3) \times 10^{-7} \mathrm{~cm}^{-2}$ above $100 \mathrm{MeV}$ with an photon index of $3.69 \pm 0.11$ (only statistical errors are given; see online supplements for a discussion of systematic errors). The high-energy component has an integral flux of $(1.3 \pm 0.1) \times 10^{-7}$ $\mathrm{cm}^{-2} \mathrm{~s}^{-1}$ above $100 \mathrm{MeV}$ with a photon index of $1.67 \pm 0.04$. Due to its hard energy spectrum the high-energy component dominates the emission above $426 \pm 35 \mathrm{MeV}$.

In order to search for flux variability of both spectral components in the LAT band, we grouped the flux measurements into monthly time bins. The high-energy component was found to be stable. The low-energy component was found to vary on these time scales (Fig. 2); the probability that the measured flux variations are statistical measurement fluctuations in a constant source is less than $10^{-5}$. No significant spectral variations were detected for either component on monthly time scales. Flux variability was also searched for on sub-monthly time scales, for which the low-energy component of the nebula is significantly detected by the LAT only in high-flux states. The flux of the low-energy component was significantly enhanced compared to the average values in February 2009 and September 2010 (Fig. 2). No variations were found for the high-energy component. The September flare was first announced by the AGILE gamma-ray mission (22), which additionally reports a flare in October 2007, before the start of Fermi observations (23). The Fermi-LAT detected flare in February 2009 was not detected by AGILE as the instrument was pointing at a different part of the sky.

The February flare had a duration of $\sim 16$ days. The average integral flux above $100 \mathrm{MeV}$ of the low-energy component between MJD 54857.73 and 54873.73 was $(23.2 \pm 2.9) \times 10^{-7}$ $\mathrm{cm}^{-2} \mathrm{~s}^{-1}$, corresponding to an increase by a factor $3.8 \pm 0.5$ compared with the average value; the increase is significant at $>8-\sigma$ level. The September flare lasted for only $\sim 4$ days. The integral flux above $100 \mathrm{MeV}$ between MJD 55457.73 and 55461.73 was $(33.8 \pm 4.6) \times 10^{-7}$ $\mathrm{cm}^{-2} \mathrm{~s}^{-1}$, corresponding to an increase by a factor $5.5 \pm 0.8$ with respect to the average and a significance of $>10 \sigma$.

The February flare has a soft spectrum with a photon index of $4.3 \pm 0.3$ (Fig. 1). The spectral slope is compatible with the average 25 -month value within two standard deviations. The energy spectrum for the second flare was significantly harder, with a photon index of 2.7 
\pm 0.2 , and was still detected above $1 \mathrm{GeV}$ at a $3-\sigma$-level. The average power released in each of the gamma-ray flares was approximately $4 \times 10^{36} \mathrm{erg} \mathrm{s}^{-1}$, for the case of isotropic emission. No significant variations in the emission of the pulsar were detected on monthly and four-day time scales through the period of observations. Examination of the timing residuals of the pulsed emission indicated no significant variations during either flares nor any significant glitch activity during the first 25 months of LAT observations.

No variations in the synchrotron component between infrared and X-ray frequencies were seen about the average nebular flux level during the second flare (24). We analyzed data collected by the BAT instrument on board the Swift satellite (25), which continuously monitors the sky at photon energies of $15-150 \mathrm{keV}$. The mean flux measured during the first flare was $(2.0$ $\pm 0.1) \times 10^{-8} \mathrm{erg} \mathrm{cm}^{-2} \mathrm{~s}^{-1}$, the flux during the second flare was $(2.0 \pm 0.1) \times 10^{-8} \mathrm{erg} \mathrm{cm}^{-2}$ $\mathrm{s}^{-1}$. Both observations are therefore within $5 \%$ of the average flux of $(2.09 \pm 0.10) \times 10^{-8} \mathrm{erg}$ $\mathrm{cm}^{-2} \mathrm{~s}^{-1}$ measured by BAT in this energy range (26), and show no correlation to the gammaray flares. The angular resolution of the BAT only allows for the measurement of the spatially integrated spectrum. Sub-arc-second resolution images were taken in X-rays by the Chandra observatory and optical by the Hubble Space Telescope a few days after the second flare. Although both images show no unusual activity compared to previous observations, both show a brightening $3^{\prime \prime}$ east of the pulsar (27). In the Chandra image this brightening is associated with a knot of $\sim 1^{\prime \prime}$ diameter that might be associated with the inner ring or the base of the jet. Such a brightening might be interpreted as an afterglow at lower frequencies of the gamma-ray flare, but no conclusions can be drawn based on one event.

The brief flare time scales and the requirement that the emission volume be causally connected imply that the flaring region must have been compact. If $L$ is the diameter of the flaring region along the line-of-sight and $t$ is the flare duration, then $L<D c t$, where the Doppler factor $D$ accounts for relativistic boosting effects. The Doppler factor is expected to be moderate within the Crab Nebula, as the typical velocities observed are smaller than $0.9 c$ (7). Even if the emission region was moving directly toward us, this yields $D<4.4$. For a flare duration of 4 days this results in $L<1.4 \times 10^{-2} \mathrm{pc}$, which corresponds to $<1.5^{\prime \prime}$ projected on the sky. Structures this small are found only in the inner part of the nebula, close to the termination shock, the base of the jet or the pulsar, suggesting that the gamma-ray emission detected in the flare originated from these regions. This is in agreement with expectations of relativistic magnetohydrodynamic simulations, in which the gamma-ray emission of the synchrotron component originates close to the termination shock $(28,29)$.

The extrapolation of the the LAT spectrum of low-energy component to lower frequencies suggest that it represents synchrotron emission (Fig. 1). The brevity of the gamma-ray flares strengthens this scenario: If the flare were instead produced by IC radiation or Bremsstrahlung, the cooling time of the emitting electrons would greatly exceed the flare duration. The cooling via Bremsstrahlung in particle densities $<10 \mathrm{~cm}^{-3}$ (30) happens over $\sim 10^{6}$ years. Similarly, electrons cooling via IC emission of $100 \mathrm{MeV}$ gamma rays on the photons of the synchrotron component of Crab Nebula have cooling times $\gtrsim 10^{7}$ years. The average magnetic field inside the Crab Nebula is estimated to be $\sim 200 \mu \mathrm{G}$, as deduced from modeling of the broad-band 
SED $(12,21)$, and might be enhanced locally by up to an order of magnitude in the inner nebula (31). These fields imply synchrotron cooling times $\lesssim 15$ days, comparable to the flare duration, leaving synchrotron radiation as the only plausible process responsible for the gamma-ray emission during the flares.

The detection of synchrotron photons up to energies of $>1 \mathrm{GeV}$ confirms that electrons are accelerated to energies of $\gtrsim 1 \mathrm{PeV}$ in the Crab Nebula (32). These are the highest energy particles that can be associated directly with any astronomical source, and they pose special challenges to particle acceleration theory. Because synchrotron losses are so efficient, there must be a strong electric field $E$ to compensate radiation reaction, given by:

$$
E / B \approx r_{\mathrm{L}} / \ell_{\mathrm{cool}} \gtrsim\left(1.3 \alpha \mathcal{E}_{\gamma \mathrm{pk}} / m_{e} c^{2}\right) \approx\left(\mathcal{E}_{\gamma \mathrm{pk}} / 50 \mathrm{MeV}\right)
$$

where $r_{\mathrm{L}}$ is the Larmor radius, $\ell_{\text {cool }}$ is the radiative cooling length, $\alpha$ is the fine structure constant and $\mathcal{E}_{\gamma \mathrm{pk}}$ is the peak synchrotron frequency at which the most energetic electrons are emitting $(17,33)$. Due to the detection of gamma-ray emission beyond $1 \mathrm{GeV} \mathcal{E}_{\gamma \mathrm{pk}}$ can be conservatively estimated to be greater than $200 \mathrm{MeV}$. The electric field is unlikely to exceed the magnetic field; if it did, there would be a local reference frame with a pure electric field in which vacuum breakdown would occur quickly. We conclude that the electric field, as measured in the Crab frame, is close in magnitude to the magnetic field in the region where the highest energy synchrotron photons were emitted. This subsumes the possibility of bulk relativistic motion. Furthermore, the resistive force due to radiation reaction is competitive with the Lorentz force and the cooling length is comparable with the Larmor radius. This poses severe difficulties to the widely-discussed acceleration mechanism of diffusive shock acceleration $(34,35)$. The proposed acceleration due to absorption of ion cyclotron waves does not suffer from these constraints (36). However, it appears to operate on time scales which are too long to accommodate the fast variability seen during the flares. Alternatively, the acceleration could be related directly to the electric field from the pulsar.

The Crab Nebula is powered by the central neutron star which acts as a DC unipolar inductor and a source of an $\mathrm{AC}$ striped wind $(2,3)$. What happens to the DC and AC current flows is controversial. It is widely supposed that $\sim 90 \%$ of the DC current returns in an outflowing wind that becomes particle-dominated and encounters a (mostly invisible) termination shock at a radius $\sim 0.1 \mathrm{pc}(37)$, but the wind could also remain electromagnetically dominated $(33,38)$. For the measured spin-down rate, a moment of inertia of $\sim 1 \times 10^{45} \mathrm{~g} \mathrm{~cm}^{2}$ and a force-free model of the magnetosphere, the total induced potential difference is $\sim 50 \mathrm{PV}$, high enough to accelerate particles to the required energies. The current associated with this potential is $\sim 300$ TA yielding a DC power per hemisphere of $\sim 1.5 \times 10^{38} \mathrm{erg} \mathrm{s}^{-1}$, a factor $\sim 40$ larger than the power released in the flares. Another interesting possibility is that particle acceleration takes place in the AC striped wind of the pulsar due to magnetic reconnection, although it is not clear if this process can accelerate particles to $\mathrm{PeV}$ energies on the required time scales $(39,40)$.

The observations reported here have raised compelling questions on our understanding of particle acceleration and motivate more detailed calculations; together with the ongoing gammaray observations of the LAT and observational campaigns at X-ray and optical wavelengths they 
might soon pinpoint the gamma-ray emission site in the Crab Nebula. 


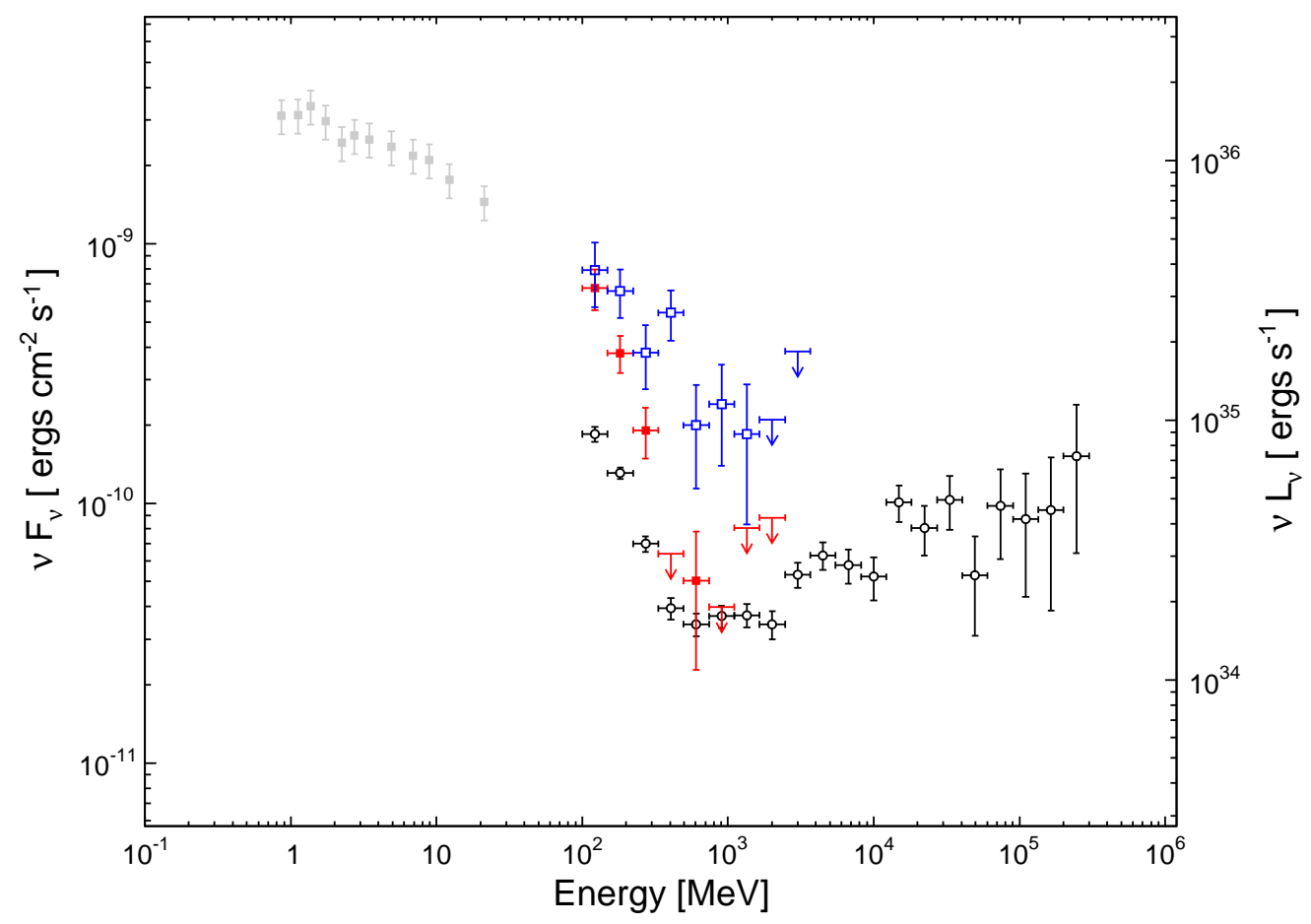

Figure 1: Spectral energy distribution of the Crab Nebula. Black open circles show the average spectrum measured by the LAT in the first 25 months of observations. Red squares show the energy spectrum during the flare of February 2009 (MJD 54857.73-54873.73) and blue open squares the spectrum in September 2010 (MJD 55457.73-55461.73). Gray squares show historical long-term average spectral data from the COMPTEL telescope with $15 \%$ systematic errors (41). Arrows indicate 95\% confidence flux limits. 


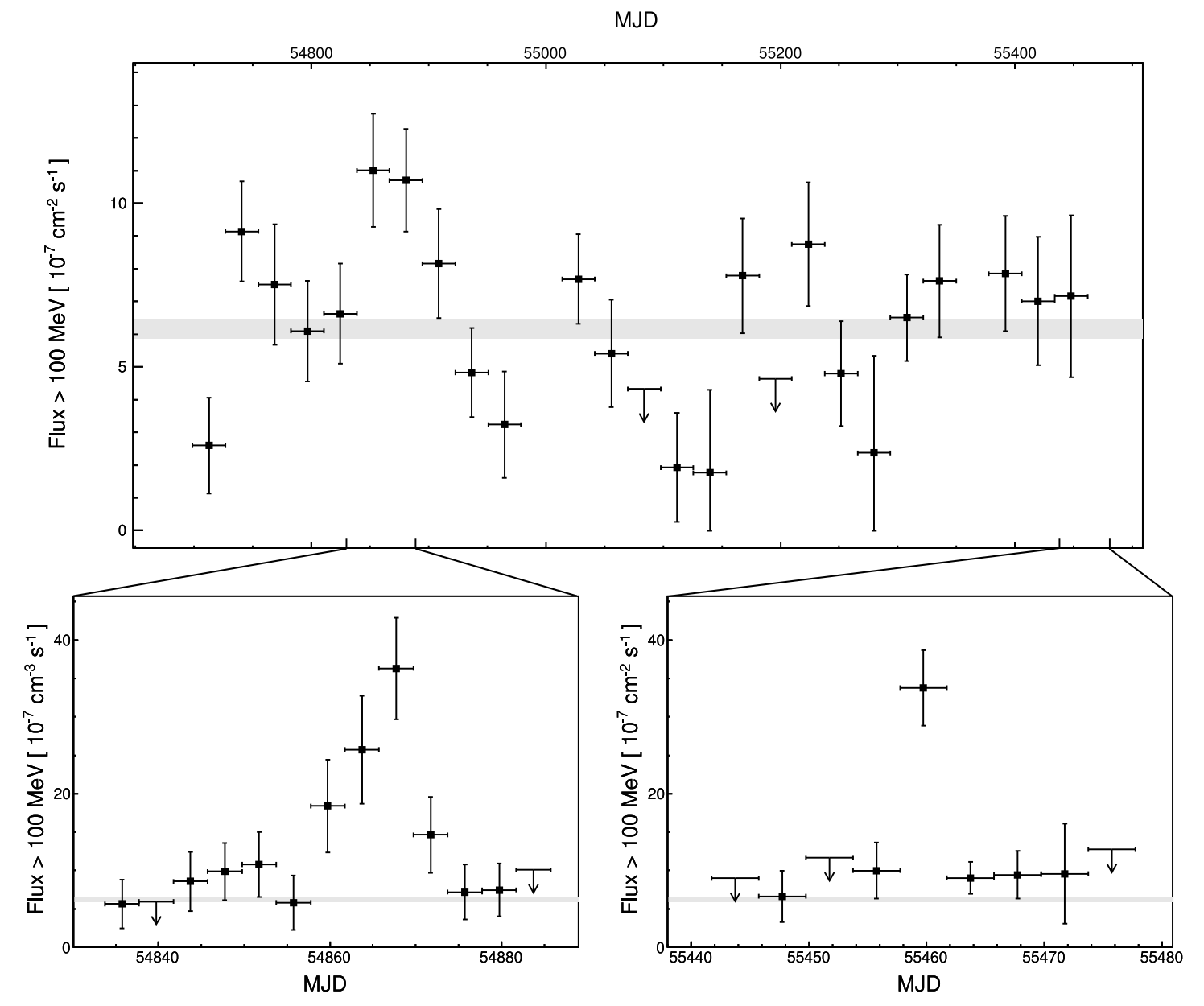

Figure 2: Gamma-ray flux above $100 \mathrm{MeV}$ as a function of time of the synchrotron component of the Crab Nebula. The upper panel shows the flux in four-week intervals for the first 25 month of observations. Data for times when the sun was within $15^{\circ}$ of the Crab Nebula have been omitted. The gray band indicates the average flux measured over the entire period. The lower panel shows the flux as a function of time in four-day time bins during the flaring periods in February 2009 and September 2010. Arrows indicate 95\% confidence flux limits. 


\section{References and Notes}

1. V. Trimble, Publications of the Astronomical Society of the Pacific 85, 579 (1973).

2. M. J. Rees, J. E. Gunn, Monthly Notices of the Royal Astronomical Society 167, 1 (1974).

3. C. F. Kennel, F. V. Coroniti, The Astrophysical Journal 283, 710 (1984).

4. J. J. Hester, Annual Review of Astronomy and Astrophysics 46, 127 (2008).

5. M. C. Weisskopf, et al., The Astrophysical Journal 536, L81 (2000).

6. J. D. Scargle, The Astrophysical Journal 156, 401 (1969).

7. J. J. Hester, et al., The Astrophysical Journal 577, L49 (2002).

8. W. J. Cocke, M. J. Disney, G. W. Muncaster, Nature 227, 1327 (1970).

9. R. Novick, M. C. Weisskopf, R. Berthelsdorf, R. Linke, R. S. Wolff, The Astrophysical Journal 174, L1 (1972).

10. A. J. Dean, et al., Science 321, 1183 (2008).

11. R. J. Gould, G. R. Burbidge, Annales d'Astrophysique 28, 171 (1965).

12. A. M. Atoyan, F. A. Aharonian, Monthly Notices of the Royal Astronomical Society 278, 525 (1996).

13. M. C. Weisskopf, et al., The Astrophysical Journal 713, 912 (2010).

14. M. Meyer, D. Horns, H. S. Zechlin, The crab nebula as a standard candle in very highenergy astrophysics, http://adsabs.harvard.edu/abs/2010arXiv1008.4524M (2010).

15. C. A. Wilson-Hodge, et al., When a standard candle flickers, http://adsabs.harvard.edu/abs/2010arXiv1010.2679W (2010).

16. R. Much, et al., Astronomy and Astrophysics 299, 435 (1995).

17. O. C. de Jager, et al., The Astrophysical Journal 457, 253 (1996).

18. F. Aharonian, et al., The Astrophysical Journal 614, 897 (2004).

19. F. Aharonian, et al., Astronomy and Astrophysics 457, 899 (2006).

20. J. Albert, et al., The Astrophysical Journal 674, 1037 (2008).

21. A. A. Abdo, et al., The Astrophysical Journal 708, 1254 (2010). 
22. Astronomer's telegram: 2855, http://www.astronomerstelegram.org (2010).

23. M. Tavani et al., Discovery of powerful gamma-ray flares from the crab nebula, Published in this journal (2010).

24. Astronomer's telegrams: 2867, 2872, 2893, http://www.astronomerstelegram.org (2010).

25. M. Ajello, et al., The Astrophysical Journal 673, 96 (2008).

26. BAT digest, http://heasarc.gsfc.nasa.gov/docs/swift/analysis/bat_digest.html.

27. Astronomer's telegrams: 2882, 2903, http://www.astronomerstelegram.org (2010).

28. D. Volpi, L. D. Zanna, E. Amato, N. Bucciantini, Astronomy and Astrophysics 485, 337 (2008).

29. S. S. Komissarov, M. Lyutikov, On the origin of variable gamma-ray emission from the Crab Nebula http://adsabs.harvard.edu/abs/2010arXiv1011.1800K (2010)

30. A. M. Atoyan, F. A. Aharonian, Astronomy and Astrophysics Supplement Series 120, 453 (1996).

31. J. J. Hester, et al., The Astrophysical Journal 448, 240 (1995).

32. O. C. de Jager, A. K. Harding, The Astrophysical Journal 396, 161 (1992).

33. M. Lyutikov, Monthly Notices of the Royal Astronomical Society 405, 1809 (2010).

34. Y. A. Gallant (2002), Relativistic Flows in Astrophysics, Lecture Notes in Physics-/, A. W. Guthmann, M. Georganopoulos, A. Marcowith, K. Manolakou, ed. (2002), 589, 24 (2002)

35. L. Sironi, A. Spitkovsky, The Astrophysical Journal 698, 1523 (2009).

36. E. Amato, J. Arons, The Astrophysical Journal 653, 325 (2006).

37. N. Bucciantini, J. Arons, E. Amato, Monthly Notices of the Royal Astronomical Society, $1423(2010)$

38. R. D. Blandford, Lighthouses of the Universe: The Most Luminous Celestial Objects and Their Use for Cosmology, M. Gilfanov, R. Sunyeav, \& E. Churazov, ed. (2002), 381

39. Y. Lyubarsky, M. Liverts, The Astrophysical Journal 682, 1436 (2008).

40. W. Bednarek, W. Idec, http://adsabs.harvard.edu/abs/2010arXiv1011.4176B (2010)

41. L. Kuiper, et al., Astronomy and Astrophysics 378, 918 (2001). 
42. Fermi science support center, http://fermi.gsfc.nasa.gov/ssc/data/analysis/scitools/overview.html.

43. A. A. Abdo, et al., The Astrophysical Journal Supplement Series 188, 405 (2010).

44. Astronomer's telegram 2872, http://www.astronomerstelegram.org (2010).

45. The Fermi LAT Collaboration acknowledges support from a number of agencies and institutes for both development and the operation of the LAT as well as scientific data analysis. These include NASA and DOE in the United States, CEA/Irfu and IN2P3/CNRS in France, ASI and INFN in Italy, MEXT, KEK, and JAXA in Japan, and the K. A. Wallenberg Foundation, the Swedish Research Council and the National Space Board in Sweden. Additional support from INAF in Italy and CNES in France for science analysis during the operations phase is also gratefully acknowledged. 


\section{Supplements}

\section{Fermi-LAT Analysis}

The LAT analysis presented in this publication was performed using the Science Tools v9r17p0 with the P6_V3_DIFFUSE instrument response functions (42). Fluxes were estimated by maximizing the likelihood of a given source model using the unbinned gtlike tool. The model was composed of the Galactic and isotropic diffuse models (v02 (43)), as well as all sources within $15^{\circ}$ of the Crab pulsar in the first Fermi LAT catalog (1FGL (43)). Only photons within this same radius and with energies greater than $100 \mathrm{MeV}$ were considered. All data taken under optimal conditions between MJD 54684.73 and 55477.73 were considered, except for periods when the sun was within $15^{\circ}$ of the Crab Nebula. To avoid background contamination from the gamma-ray emission of the earth limb only time periods where the Crab Nebula was at a zenith angle $<90^{\circ}$ were considered. In order to avoid the strong foreground emission of the pulsar, only rotational phases between 0.52 and 0.87 were considered for the nebula analysis (21). The pulsar timing model used will be made available through the Fermi Science Support Center (42).

Light curves were generated by performing the likelihood fit in each time bin independently. The free parameters in the 28-day likelihood fits were the fluxes and spectral indices of the synchrotron and IC components of the nebula and the amplitude of the Galactic and isotropic diffuse emission. All other parameters were held fixed to their 1FGL values. In the 4-day light curves, the only free parameters of the likelihood fits were the flux and spectral index of the synchrotron component. The IC flux and spectral index and the Galactic and isotropic diffuse normalizations were held fixed to their 25 -month best-fit values. Spectral data points on the SED were measured by performing the likelihood fit in each energy bin independently, varying only the normalization of the best-fit model. For both light curves and energy spectra, 95\% confidence upper limits on the flux were derived if the significance of the source detection was below $2 \sigma$.

\section{Significance and position of the flares}

The flaring periods were identified by fitting a flaring component together with the average 25month spectral model in weekly time bins between MJD 54684.73 and 55461.73. The average model included the two components of the Crab Nebula. The flaring component was modeled by a power-law energy spectrum and its normalization and index were the only parameters varied in the fits. The significance of the flaring component was $<3.5 \sigma$ for all weeks outside of the flare periods, while it was $>6.7 \sigma$ during the flares. Considering trials for the 102 analyzed weeks this corresponds to significances $<2$ and $>6 \sigma$ respectively. To measure the properties of the emission during the flare in more detail two flaring periods were defined from the 4 day light curves shown in Figure 2. For the first flare the flaring period was defined as MJD 54857.73-54873.73 (the four high flux bins around the flare). Fitting the additional source with the average best-fit model yields a significance of $>8 \sigma$ for the flaring component. For the 
second flare the flaring period was defined as MJD 55457.73-55461.73. The significance of the flaring component during this time period is $>10 \sigma$.

For both flares the position of the flaring component was derived using the gtfindsrc program of the Science Tools. Both positions are compatible with the Crab Nebula. The best-fit position of the first flare is R.A.: $83.73^{\circ}$, Dec: $21.81^{\circ}$ with a $68 \%$ containment radius of $0.22^{\circ}(\mathrm{J} 2000)$, one standard deviation away from the Crab pulsar position of R.A.: $83.63^{\circ}$ Dec: $22.01^{\circ}$. The best-fit position of the second flare is R.A.: $83.68^{\circ}$, Dec: $22.03^{\circ}$ with a $68 \%$ containment radius of $0.044^{\circ}, 1.1 \sigma$ away from the pulsar.

\section{The possibility of a background blazar}

The monthly flux variability and flaring periods in principle might be due to the emission of a background source that cannot be resolved within the angular resolution of the LAT. However, this scenario is very unlikely for several reasons. First, the flux increase during both flares above $100 \mathrm{MeV}$ was $\gtrsim 2 \times 10^{-6} \mathrm{~cm}^{-2}$; there are fewer than 100 LAT sources which show variability of this magnitude. The probability of chance coincidence for one of them to be located behind the Crab Nebula within the $2 \sigma$ localization error measured for the second flare, is $<6 \times 10^{-5}$. Second, the spectral index during the first flare is the softest of any source yet detected by the LAT, with the exception of the synchrotron component of the Crab Nebula itself $(21,43)$, suggesting that the emission originate from the same source (this argument also relates the second flare to the Crab Nebula, as the probability that both flares are produced by two different sources is negligible, due to the even lower chance probability of positional coincidence in this case). Finally, the only source class known to produce variability on the flare time scales of the observed magnitude in gamma-rays are blazars. No known blazar is located near the Crab Nebula within the angular resolution of Fermi and X-ray observations taken two days after the second flare revealed no new source which might be associated with a yet-unknown blazar (44).

\section{Systematic Uncertainties}

Stability of flux measurements with the LAT data over time was tested using sources which are expected to be constant in flux. These sources are the Crab pulsar, the nearby Geminga pulsar, the Vela pulsar and the Galactic diffuse emission. We found the light curve of each of these reference sources was consistent with a constant on monthly and 4-day timescales if we added a 5\% systematic error in quadrature with the statistical errors. We have therefore added a 5\% systematic error in quadrature to all light curves in this publication and have taken this systematic error to be time-variable and normally distributed. We adopt $30 \%$ as the systematic uncertainty for overall normalization of integrated fluxes $>100 \mathrm{MeV}$ reported here. The errors given on LAT spectral points are statistical only. The uncertainties in the energy dependence of

the acceptance and energy disperson of the LAT leads to a systematic error of 0.1 on the photon index (21). 